\title{
Plasma miRNA-155 Levels Predict Atrial Fibrillation Recurrence after Cardioversion
}

\author{
Xiong Zhang, ${ }_{1}^{1}$ Xiao-ping Xiao, ${ }^{2} \mathrm{Xu}-\mathrm{ai}$ Ren, BM, ${ }^{3}$ Tao Cui, $\mathrm{BM}^{3}$ \\ ${ }^{1}$ Department of Laboratory, the First People's Hospital of Xianyang City, Xianyang, Shaanxi Province, China; ${ }^{2}$ Department of \\ Laboratory, the Eighth Hospital of Xi'an City, Xi'an, Shaanxi Province, China; ${ }^{3}$ Department of Cardiology, the First People's \\ Hospital of Xianyang, Xianyang, Shaanxi Province, China
}

\section{ABSTRACT}

Background: MicroRNAs (miRNAs) are widely involved in the regulation of physiological processes, such as cell proliferation, differentiation, apoptosis, angiogenesis, and lipid metabolism. They might be associated with the pathological process of atrial fibrillation (AF). The purpose of our study is to investigate whether plasma miRNA-155 levels have a relationship with $\mathrm{AF}$ recurrence.

Methods: A total of 110 patients with AF were studied, all with successful cardioversion. We measured the expression of plasma miRNA-155 in the recurrent group $(\mathrm{n}=30)$ and in the nonrecurrent group $(n=80)$ by quantitative reverse transcription-polymerase chain reaction (qRT-PCR). In addition, the serumal levels of B-type natriuretic peptide (BNP), total cholesterol (TC), and fasting blood glucose (FBG) in the groups were determined by using an automatic biochemical analyzer; and an immunoenzymatic method was applied to determine the serumal levels of tumor necrosis factor- $\alpha$ (TNF- $\alpha$ ), C-reactive protein (CRP), and interleukin-6 (IL-6). The left atrial diameter (LAD) and left ventricular ejection fraction (EF) of all patients were measured by using echocardiography.

Results: Our RT-PCR analysis found that miRNA-155 was significantly upregulated in the recurrent group compared with the nonrecurrent group. These increases of LAD and the levels of BNP, TNF- $\alpha$, CRP, and IL-6 in the recurrent group were also revealed to be relative to those in the nonrecurrent group. There were no differences in the levels of TC and

Received November 4, 2018; accepted December 26, 2018.

Abbreviations: $A F$, atrial fibrillation; $A U C$, area under the curve; $B N P, B$-type natriuretic peptide; CAD, coronary artery disease; CRP, C-reactive protein; $C T$, changes of cycle threshold; DM, diabetes mellitus; DSS, dextran sulphate sodium; EF, left ventricular ejection fraction; FBG, fasting blood glucose; bsCRP, bigh-sensitivity C-reactive protein; IL-6, interleukin-6; LAD, left atrial diameter; LPS, lipopolysaccharide; miRNA, microRNA; MVD, mitral valve disease; PAF, paroxysmal atrial fibrillation; qRT-PCR, quantitative reverse transcription-polymerase chain reaction; $R F U$, relative fluorescence unit; $R O C$, receiver operating characteristic; $S D$, standard deviation; TC, total cholesterol; $T N F-\alpha$, tumor necrosis factor- $\alpha$.

Correspondence: Tao Cui, Department of Cardiology, First People's Hospital of Xianyang, No. 10 Biyuan West Road, Xianyang City, Shaanxi Province 712000,China; +86-15706030285 (e-mail:280310216@qq.com).
FBG, as well as in EF, between the groups. Moreover, miRNA155 expression was observed to correlate positively with these outcomes of LAD, BNP, TNF- $\alpha$, CRP, IL-6, and LAD. A diagnostic significance of predicting $\mathrm{AF}$ recurrence for plasma miRNA-155 was elucidated via ROC curve analysis.

Conclusions: Our findings revealed that plasma miRNA-155 can present an ability to calculate $\mathrm{AF}$ recurrence after cardioversion.

\section{BACKGROUND}

Atrial fibrillation (AF) is the most common clinical arrhythmia, affecting approximately 33.5 million people annually worldwide [Chugh 2014]. Its morbidity has increased with the aging of the general population [Kannel 1998; Naccarelli 2009]. Atrial remodeling is characterized by several atrial structural and functional alterations that occur as a consequence of AF. These changes can be classified as electrical remodeling, tissue (structural) remodeling, $\mathrm{Ca}^{2+}$-handling abnormalities, and neurohormonal changes [Nattel 2014]. In nonparoxys$\mathrm{mal} \mathrm{AF}$, atrial structure remodeling has been thought to be associated with atrial enlargement, ultrastructure changes in

Table 1. Baseline Clinical Characteristics of the AF Patients*

\begin{tabular}{lccc}
\hline & $\begin{array}{c}\text { Nonrecurrent } \\
\text { Group }(\mathrm{n}=80)\end{array}$ & $\begin{array}{c}\text { Recurrent } \\
\text { Group }(\mathrm{n}=30)\end{array}$ & $P$ \\
\hline Age, years, mean $\pm \mathrm{SD}$ & $59.03 \pm 13.29$ & $60.72 \pm 13.63$ & .897 \\
Male, $\mathrm{n}(\%)$ & $49(61.3 \%)$ & $17(56.6 \%)$ & .669 \\
Duration of AF, years, mean $\pm \mathrm{SD}$ & $2.0 \pm 2.6$ & $2.1 \pm 2.5$ & $<.01$ \\
Persistent AF, n (\%) & $33(41.2 \%)$ & $20(66.6 \%)$ & .020 \\
Paroxysmal AF, n (\%) & $47(58.8 \%)$ & $10(33.4 \%)$ & .020 \\
Hypertension, $\mathrm{n}(\%)$ & $40(50.0 \%)$ & $16(53.3 \%)$ & .832 \\
DM, n (\%) & $17(21.2 \%)$ & $8(26.6 \%)$ & .612 \\
CAD, n (\%) & $25(31.2 \%)$ & $11(36.6 \%)$ & .651 \\
MVD, n (\%) & $3(3.7 \%)$ & $5(16.6 \%)$ & .034 \\
Hyperlipidemia, n (\%) & $35(43.7 \%)$ & $16(53.3 \%)$ & .398 \\
\hline
\end{tabular}

*AF, atrial fibrillation; SD, standard deviation; DM, diabetes mellitus; CAD, coronary artery disease; MVD, mitral valve disease. 
Table 2. Primers Used in qPCR Experiments*

\begin{tabular}{lc}
\hline miRNA & Primer Sequence $\left(5^{\prime}-3^{\prime}\right)$ \\
miRNA-155 & Forward, TTAATGCTAATCGTGA \\
& Reverse, ACCTGAGAGTAGACCAGA \\
3 -actin & Forward, ATGTCACGCACGATTTCC \\
& Reverse, CTGTCCCTGTATGCCTCTG \\
\hline
\end{tabular}

*qPCR, quantitative polymerase chain reaction; miR-155, microRNA-155.

cardiac cells , and atrial interstitial fibrosis [Pison 2016]. Notably, absolute AF burden has been defined as the average time (hours) of AF duration over the whole observational period. Available evidence suggests that the altered oxidative stress stimulating the activation of multiple cytokines and inflammatory pathways seems to influence $\mathrm{AF}$ burden in response to catheter ablation treatment [Sardu 2017]. Accordingly, AF frequently recurs after ablation treatment [Mitrić 2016]. A recent analysis has indicated that subclinical episodes of $\mathrm{AF}$ occur frequently in type 2 diabetic patients and are associated with a significantly increased risk of silent cerebral infarct and stroke [Marfella 2013]. Oral anticoagulation therapy is the treatment of choice for preventing thromboembolism diseases caused by AF. However, only $46.2 \%$ of patients with AF take oral anticoagulation medications [Genovesi 2015].

Therefore, appropriate prediction contributes to the early identification of patients at a high risk of AF recurrence and is conducive to determine a suitable therapeutic regimen at the early stage and to reduce the patient risk and medical expense.

MicroRNAs (miRNAs) are small noncoding endogenous RNAs, which have been used as the biomarkers of fibrosis and electrical alteration for $\mathrm{AF}$, and have become the targets of selective therapy for $\mathrm{AF}$.

Moreover, catheter ablation can electrically isolate the pulmonary vein, which can be upgraded by miRNAs' therapy and prevent both cardiac electrical and fibrotic remodeling of $\mathrm{AF}$ [Sardu 2015]. The above evidence shows that miRNAs might be involved in AF pathogenesis. It has been reported that the expression of miRNAs, including miRNA-155, miRNA$146 \mathrm{~b}-5 \mathrm{p}$, and miRNA-19b, elevates significantly in patients with nonvalvular PAF [Wang 2015]. In this study, we used RT-PCR to detect miRNA-155 expression from patients with $\mathrm{AF}$ and explored the possible mechanisms of miRNA-155 in atrial remodeling as well as the association among miRNA155, inflammation, and $\mathrm{AF}$ recurrence, suggesting that miRNA-155 can be used as an effective warning marker and as an intervention target for $\mathrm{AF}$ recurrence after cardioversion.

\section{MATERIALS AND METHODS}

\section{General Data}

A total of 110 patients with $\mathrm{AF}$ were studied, all of whom had a successful cardioversion at the Department of Cardiology
Table 3. Patient Baseline Data $(\bar{\chi} \pm S D) *$

\begin{tabular}{lccc}
\hline Variables & $\begin{array}{c}\text { Nonrecurrent Group } \\
(\mathrm{n}=80)\end{array}$ & $\begin{array}{c}\text { Recurrent Group } \\
(\mathrm{n}=30)\end{array}$ & $P$ \\
\hline TNF- $\alpha, \mathrm{pg} / \mathrm{mL}$ & $5.5 \pm 2.3$ & $7.3 \pm 2.5$ & $<.01$ \\
$\mathrm{CRP}, \mathrm{mg} / \mathrm{L}$ & $1.5 \pm 0.5$ & $2.5 \pm 0.8$ & $<.01$ \\
$\mathrm{IL}-6, \mathrm{ng} / \mathrm{L}$ & $0.41 \pm 0.04$ & $0.52 \pm 0.05$ & $<.01$ \\
$\mathrm{TC}, \mathrm{mmol} / \mathrm{L}$ & $3.87 \pm 0.73$ & $4.03 \pm 0.71$ & .731 \\
$\mathrm{LAD}, \mathrm{mm}$ & $35.62 \pm 3.93$ & $50.89 \pm 5.33$ & $<.01$ \\
FBG, mmol/L & $4.10 \pm 0.57$ & $4.12 \pm 0.56$ & .753 \\
EF, \% & $56.23 \pm 5.62$ & $54.37 \pm 5.36$ & .772 \\
BNP, ng/L & $139.36 \pm 79.57$ & $556.21 \pm 123.87$ & $<.01$ \\
\hline
\end{tabular}

*All variables are denoted by mean \pm SD. TNF- $\alpha$, tumor necrosis factor- $\alpha$; CRP, C-reactive protein; IL-6, interleukin-6; TC, total cholesterol; LAD, left atrium diameter; FBG, fasting blood glucose; $E F$, left ventricular ejection fraction; BNP, B-type natriuretic peptide.

at the First People's Hospital of Xianyang City or the Eighth Hospital of Xi'an City from March 2015 to March 2017. The age of the patients (mean $\pm \mathrm{SD}$ ) was $60.12 \pm 13.37$ years. AF was paroxysmal in 57 patients and persistent in 53 patients. Recurrence in 30 patients and no recurrence in 80 patients were identified on the basis of electrocardiogram and dynamic electrocardiogram. The following cases were excluded: patients without a response to amiodarone for 12 months; patients with NYHA class I or with NYHA class I transformed from NYHA class II because of the treatment with diuretics, digitalis, or other drugs; patients with atrioventricular block, hyperthyroidism, diabetes, liver or kidney dysfunction, or acute and chronic infection disease; patients with allergy to amiodarone; patients with poisoning due to digitalis.

The baseline characteristics of all participants are presented in Table 1. The presences of persistent AF and a history of MVD (reported cases included mitral valve insufficiency $\geq 2$ or of moderate severity) were associated with a higher risk for AF recurrence after ablation. These characteristics of age, gender, AF duration, hypertension, diabetes, $\mathrm{CAD}$, and hyperlipidemia did not significantly differ between the 2 groups.

\section{Methods}

All enrolled patients with AF were subjected to radiofrequency catheter ablation, and a sustained sinus rhythm was confirmed. Then, they were given amiodarone for 3 months following a regular review in which 80 patients maintained their sinus rhythm within 12 months after ablation treatment and 30 patients relapsed. The recurrence rate was $27.27 \%$.

The levels of miRNA-155 in the plasma of patients with AF were detected by using RT-PCR. A Modular P800 automatic biochemical analyzer (Roche Diagnostics, Rotkreuz, Switzerland) and diagnostic kits (Roche) were applied to the detection of the serumal concentrations of BNP, TC, and FBG. The serumal levels of TNF- $\alpha$, CRP, and IL- 6 were 

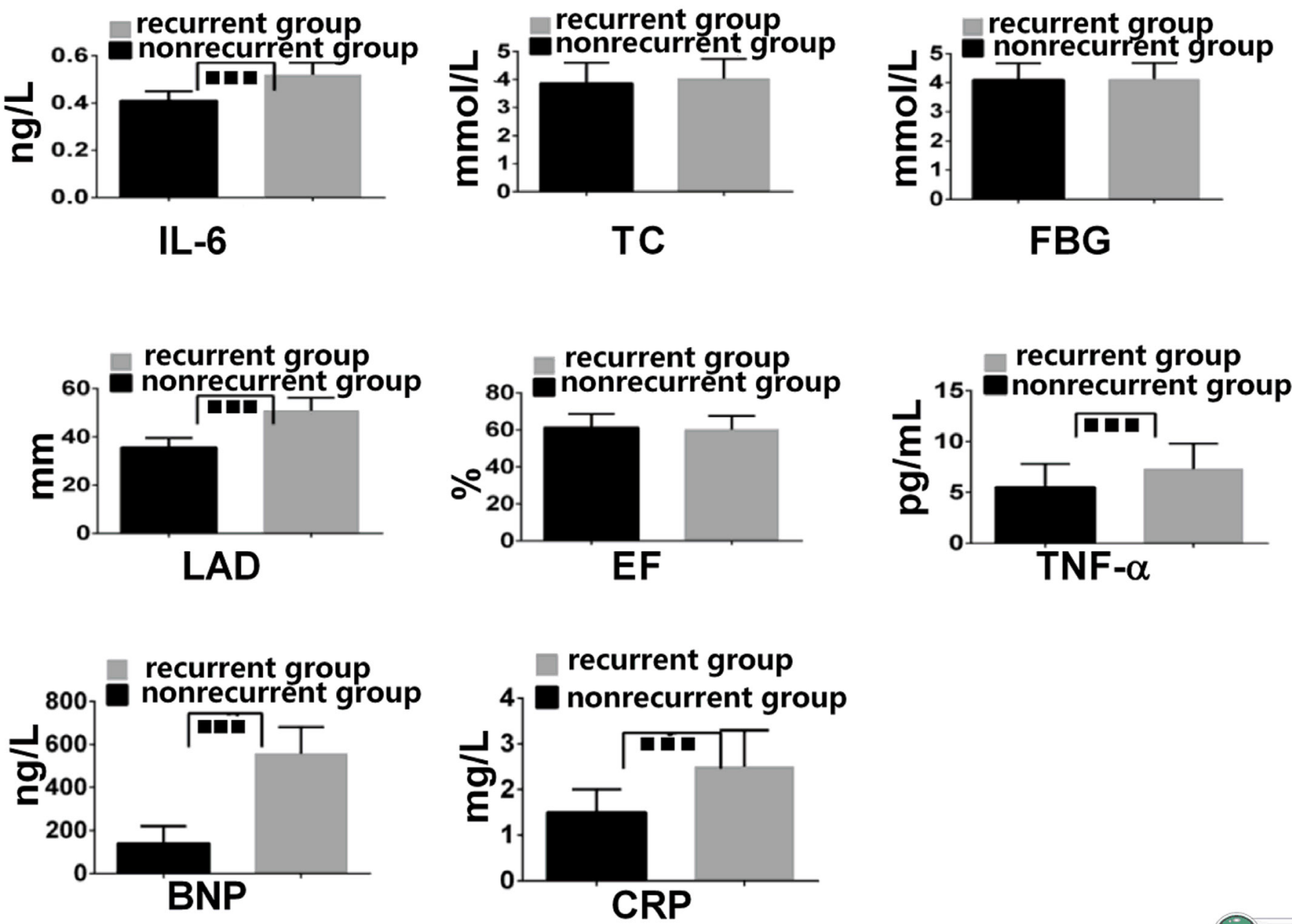

Figure 1. Validation of comparisons of baseline data between the groups. TNF- $\alpha$, tumor necrosis factor- $\alpha$; CRP, C-reactive protein; IL-6, interleukin-6; TC, total cholesterol; LAD, left atrium diameter; FBG, fasting blood glucose; EF, left ventricular ejection fraction; BNP, B-type natriuretic peptide. $\mathbf{\square} \square, P<.01$.

determined by using an immunoenzymatic method and standard laboratory techniques according to manufacturers' specifications (Quantikine ELISA kit, R\&D Systems, Minneapolis, MN, USA). All participants were subjected to color Doppler ultrasonography (Vivid7 Dimension, General Electric Company, Boston, MA, USA) that was used by measuring the LAD and EF on the day on which blood was collected.

\section{Follow-up Visits}

All the patients were followed up once each month. AF recurrence was confirmed on the basis of the patients' symptoms and the results of electrocardiogram and dynamic electrocardiogram. Within 12 months after the ablation treatment, nonrelapsing patients were assigned to the recurrent group or to the nonrecurrent group.

\section{Specimen Collection}

Six milliliters of venous blood was collected from the AF patients after a 12-month follow-up. The collected blood was placed in 2 tubes. One, which included EDTA as anticoagulant, was separated into plasma by centrifugation at a 3000-rpm speed for 15 minutes at $4^{\circ} \mathrm{C}$, with application to the measurement of miRNA-155. The other was separated serum for use in measuring the levels of BNP, FBG, TC, TNF- $\alpha, \mathrm{CRP}$, and IL-6. The separated plasma and serum were removed to freezing pipes and preserved at $-80^{\circ} \mathrm{C}$ until assayed.

\section{RNA Isolation}

Total RNA was extracted from the plasma of patients with AF by using TRIzol reagent (Invitrogen [Thermo Fisher Scientific, Waltham, MA, USA]) according to the manufacturer's protocol. RNA purity was determined in accordance with the ratio of the optical density at $260 \mathrm{~nm}$ and $280 \mathrm{~nm}$. If the optical density ratio was between 1.7 and 2.0, the RNA solution was considered pure and was immediately stored at $-80^{\circ} \mathrm{C}$.

Quantitative Reverse Transcription-Polymerase Chain Reaction

Primers (Table 2) were designed by Primer Premier 5.0 software (Premier Biosoft International, Palo Alto, CA, USA). $\beta$-Actin was used as the internal reference. The reverse transcription reaction was carried out by using a TaqMan MicroRNA Reverse Transcription Kit (Applied Biosystems 


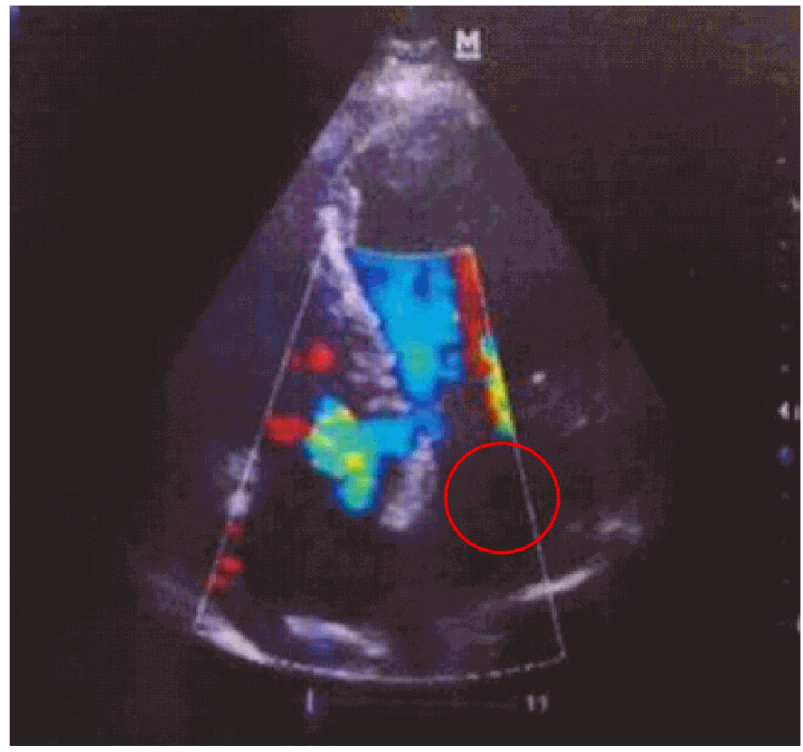

Figure 2. Evidence of left atrial dilation in patients with AF. The red, blue, yellow colors represent the blood flow signals toward the probe, the blood flow signals away from the probe, and the blood flow signals of pathological tissues, respectively. The red circle shows the enlarged atrium. AF, atrial fibrillation; M, M-mode ultrasound diagnostic instrument.

[Thermo Fisher Scientific]) according to the manufacturer's instructions. Quantitative polymerase chain reaction (qPCR) was performed by using TaqMan Universal PCR Master Mix (Applied Biosystems [Thermo Fisher Scientific]) in a 7500 Real-Time PCR system (Applied Biosystems [Thermo Fisher Scientific]) under the following conditions: $95^{\circ} \mathrm{C}$ for 3 minutes, followed by 40 cycles of $95^{\circ} \mathrm{C}$ for 15 seconds, and $60^{\circ} \mathrm{C}$ for 1 minute. The length of the amplification product for miRNA155 was $120 \mathrm{bp}$, and for $\beta$-actin, the length was $115 \mathrm{bp}$.

\section{Analysis of Gene Sequences}

The sequences of the PCR products were determined by using a sequencing machine. Sequence homology was determined by comparing the sequencing results with known sequences in GenBank by using BLAST software (NLM, NCBI, Bethesda, MD, USA).

\section{Relative Expression of miRNA}

The amplified products were analyzed by gel electrophoresis of $4 \%$ agarose. The changes of the cycle threshold (CT) were used quantitatively in determining miRNA expression. $\Delta \mathrm{CT}=(\mathrm{Xn}) \mathrm{CT}-(\beta$-actin $) \mathrm{CT}$, where $(\beta$-actin $) \mathrm{CT}$ was the CT of the internal reference and (Xn) CT was the CT of the participants. The relative expression of miRNA was calculated as $2^{-\mathrm{CT}}$.

\section{Statistical Analysis}

The significance of the experimental data was evaluated by using SPSS 21.0 statistics software (IBM Corp., Armonk, NY, USA). Continuous variables with a normal distribution

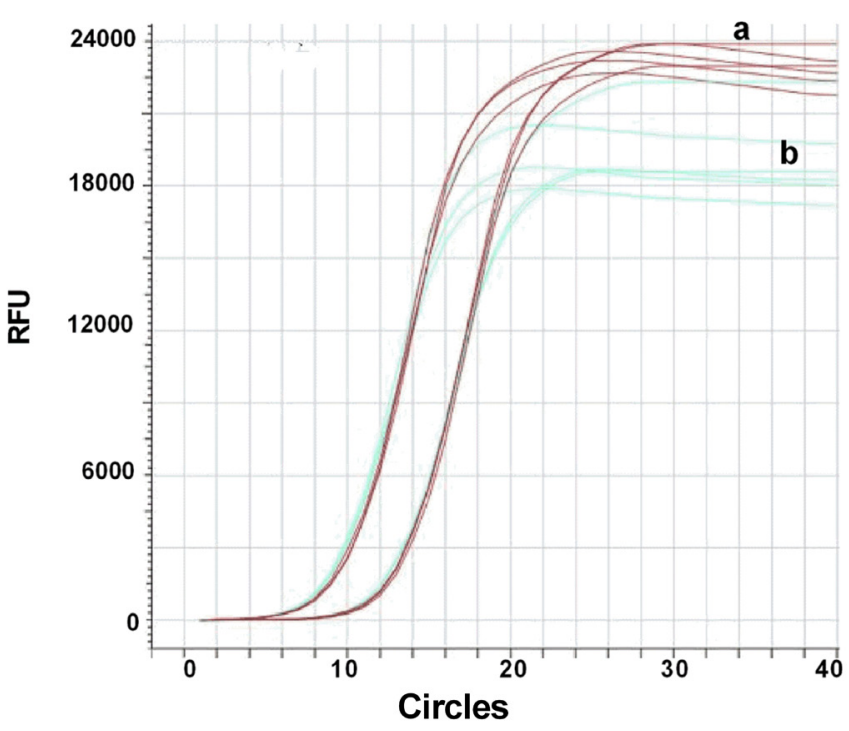

Figure 3. PCR amplification curves. The amplification curves are smooth " $S$ " shapes, showing that the operations were accurate and that the results are believable. The $a$ and $b$ (as expressed in brown and cyan lines) show the amplification curves of miRNA-155 and $\beta$-actin, respectively.

were presented as mean \pm SD and were compared by using the Student $t$ test. The categorical variables were compared via $\chi^{2}$ test. A correlation analysis was performed via the Pearson method. ROC curves were drawn, and the AUCs were measured, assessing the specificity and sensitivity of circulating miRNA-155 as diagnosing recurrence of AF. A $P$ value of less than .05 was considered significant.

\section{RESULTS}

\section{Comparison of Baseline Data between the Groups}

As shown in Table 3, these increases of the levels of BNP, TNF- $\alpha$, CRP, and IL-6 (Figure 1) and LAD (Figure 2) are observed in the recurrent group compared with in the nonrecurrent group (all $P<.01$ ). Nevertheless, the differences of EF and the levels of TC and FBG between the 2 groups of AF were not established yet (all $P>.05$ ). In Figure 1, the gray pillars, usually relatively higher in comparison with the black pillars, show these incremental levels of BNP, TNF- $\alpha$, CRP, and IL-6 in the recurrent group. Figure 2 shows the left atrial dilation in patients with AF. The red, blue, yellow colors represent the blood flow signals toward the probe, the blood flow signals away from the probe, and the blood flow signals of pathological tissues, respectively. The red circle shows an enlarged atrium.

\section{Analysis of PCR Amplification Curves}

As shown in Figure 3, the PCR amplification curves accompanying with a smooth " $\mathrm{S}$ " shape present baseline stage, logarithmic amplification stage, and platform stage, indicating the accurate operations and the believable outcomes, where 

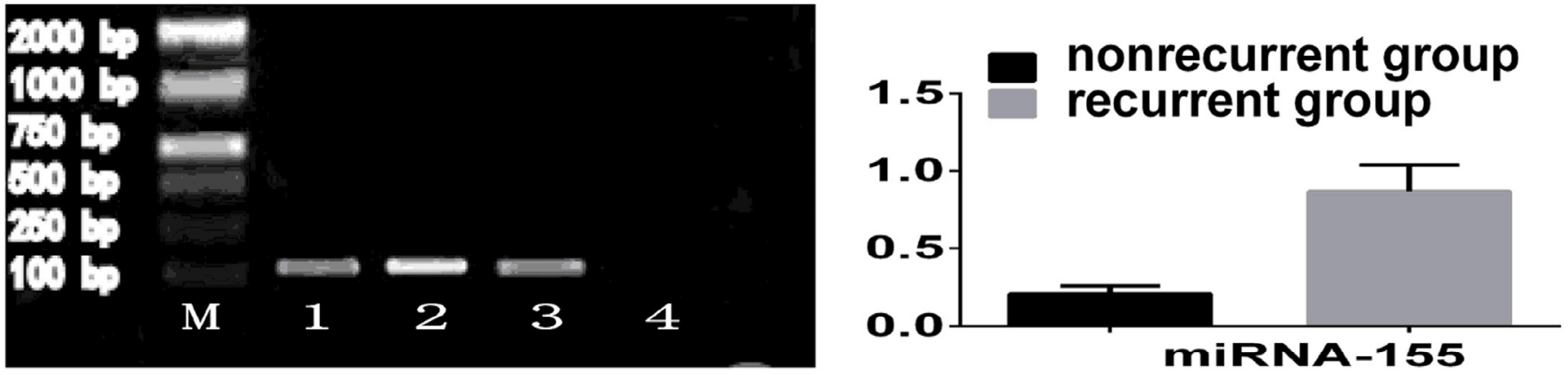

Figure 4. miRNA-155 expression. M, 1, 2, 3, and 4 show the outcomes of marker [DL 2,000 DNA Marker (Baori Medical Biotechnology Co., Ltd., Beijing, China)], miRNA-155 in the nonrecurrent group, miRNA-155 in the recurrent group, $\beta$-actin, and the negative control, respectively. The lengths of the expected miRNA-155 and $\beta$-actin were 120 bp and 115 bp, respectively. The expression of miRNA-155 was upregulated in the recurrent group compared with that in the nonrecurrent group $(P<.01)$.

curves a and b (as expressed in brown and cyan lines) represent the concentration (relative fluorescence unit [RFU]) of miRNA-155 and $\beta$-actin, respectively.

\section{Comparison of miRNA-155 Expression Levels between the Groups}

As shown in Figure 4, the expression of miRNA-155 in the recurrent and nonrecurrent groups (mean \pm SD) is 0.87 \pm 0.17 and $0.21 \pm 0.05$, respectively, confirming that miRNA155 was significantly upregulated in the recurrent group compared with the nonrecurrent group. The left figure indicates a gel electrophoresis consequence of the amplified products. The marker (M) lane has 6 bands $(100,250,500,750,1000$, and $2000 \mathrm{bp}$ ). The bands in lanes 1, 2, and 3 are between 100 and $250 \mathrm{bp}$. The band in lane 2 is significantly brighter than that in lane 1, which illustrates the increased expression of miRNA-155 in patients with recurrent AF. The bright band in lane 3 and the empty band in lane 4 confirm the accuracy of the bands in lanes 1 and 2. In the right figure, the gray pillar, which is relatively higher than the black pillar, shows an increased expression of miRNA-155 in the recurrent group.

\section{Analysis of Correlation}

As shown in Figure 5, the expression of miRNA-155 is described to be positively correlated with the levels of BNP, TNF- $\alpha$, CRP, and IL-6, as well as LAD $\left(R^{2}=0.930,0.906\right.$, $0.896,0.916,0.966$, respectively, all $P<.01)$.

\section{Analysis of Receiver Operating Characteristic Curves}

As shown in Figure 6, the green and blue lines represent the reference line and miRNA-155, respectively. The AUC of miRNA-155 is 0.888 ( $P<.01$; $95 \%$ CI: 0.808-0.969). Information from ROC curve analysis indicates that $\mathrm{AF}$ recurrence could be observed through the variant expression of miRNA155 in plasma.

\section{DISCUSSION}

Our RT-PCR analysis confirmed that miRNA-155 expression was significantly upregulated in the recurrent group compared with that in the nonrecurrent group. The analysis of the AF patients revealed the incremental presences of LAD, BNP, TNF- $\alpha$, CRP, and IL-6 for the recurrent patients with AF. There were no differences of the levels of TC, FBG, and EF between the 2 groups. Moreover, expression of miRNA155 was observed to correlate positively with the incremental presences of LAD, BNP, TNF- $\alpha$, CRP, and IL-6. ROC curve analysis ascertained that the variant expression of miRNA155 in plasma of patients with $\mathrm{AF}$ could predict $\mathrm{AF}$ recurrence after cardioversion.

\section{miRNA-155 Expression and $A F$}

Growing evidences indicates that miRNA-155 as an important pleiotropic regulator of cell homeostasis is a typical multifunctional miRNA that accommodates multiple pathophysiological processes, such as hematopoietic lineage differentiation, inflammation, immunity, viral infections, and cardiovascular diseases [van Berkel 2005; Fichtlscherer 2010]. The association between cardiovascular diseases (eg, CAD) and miRNA-155 has been increasingly studied. However, these do not give a consistent conclusion. Analysis of a highthroughput array screening for 667 miRNAs confirmed the violent expression of miRNA-155 in the serum of patients at high risk of cardiac death. Nevertheless, opposing clinical research findings in relation to miRNA-155 have been reported. miRNA-155 was negatively correlated with Th17 cell differentiation, and its downregulation was discovered in the patients with urgent coronary syndrome [Filipowicz 2008]. Briefly, both upregulation and downregulation of miRNA155 in patients with cardiovascular diseases are possible to respond to the pathological processes of these diseases. There is a previous study revealing the association between miRNA155 and arrhythmia. An analysis of 485 patients with chronic heart failure indicated that the altered regulation of miRNA155 in patients with the AT1-1166 CC genotype is predictive of an increased risk of ventricular arrhythmias [Blanco 2012].

The RT-PCR analysis of the patients with AF confirmed that the significant upregulation of miRNA-155 supplied the most pronounced change among the 10 differentially expressed miRNAs. These findings, which were obtained by analyzing the miRNA profile, indicate that miRNA-155 

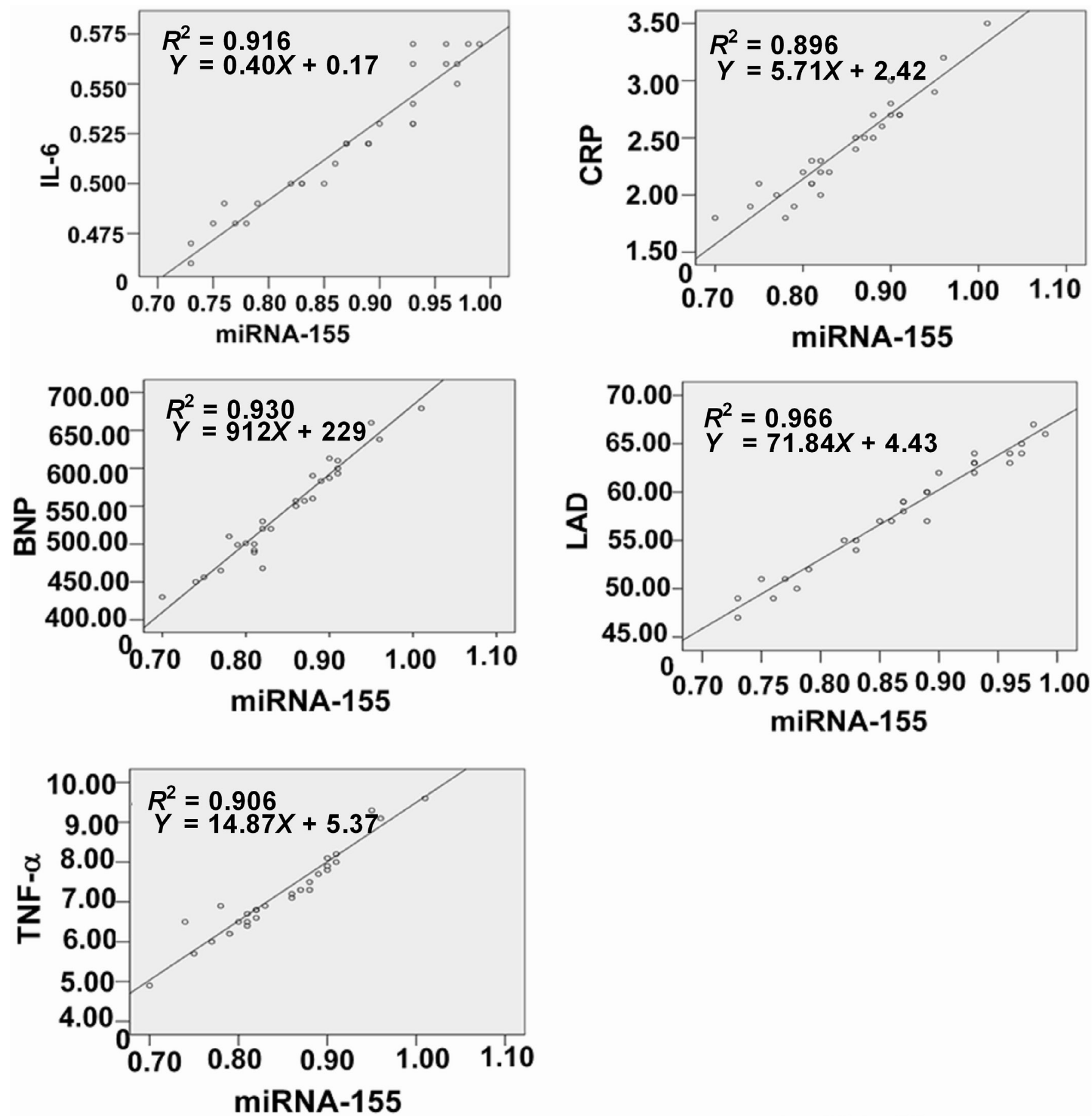

Figure 5. Correlation analysis of miRNA-155. TNF- $\alpha$, tumor necrosis factor- $\alpha$; CRP, C-reactive protein; IL-6, interleukin-6; LAD, left atrium diameter; BNP, B-type natriuretic peptide.

might have an important impact on the progression of AF. Upregulation of miRNA-155 in patients with AF is consistent with our findings. Our RT-PCR results showed that miRNA155 was remarkably higher in the recurrent group than in the nonrecurrent group. The mechanism underlying the process that miRNA-155 partakes in AF progression is known to be associated with inflammation [Wang 2015]. Recent studies have reported that some miRNAs (eg, miRNA-155 and miRNA-146a) are responsible for the regulation of inflammatory responses in macrophages and monocytes [O'Connell 2007; Hou 2009; O'Connell 2010; O'Connell 2012]. Further evidence demonstrates that inflammatory factors might 


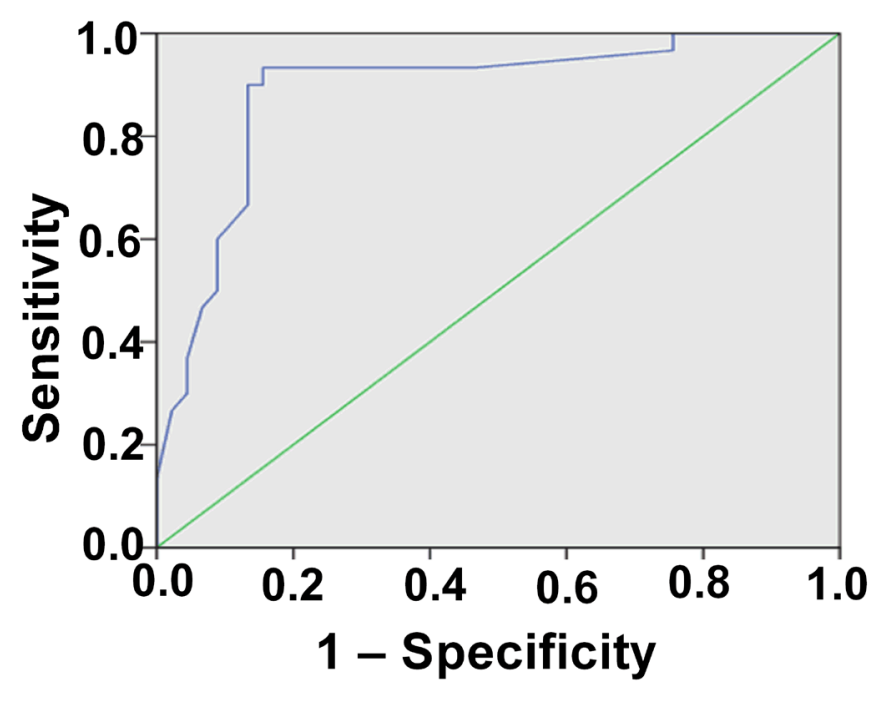

Figure 5. Correlation analysis of miRNA-155. TNF- $\alpha$, tumor necrosis factor- $\alpha$; CRP, C-reactive protein; IL-6, interleukin-6; LAD, left atrium diameter; BNP, B-type natriuretic peptide.

promote the participation of miRNA-155 in the pathological process of AF [Rebane 2013]. Nevertheless, the precise mechanism that inflammation contributes to development of AF remains unclear [Aviles 2003; Kannel 2009]. These findings suggest that the inflammatory markers, such as TNF- $\alpha$, CRP, and IL-6, have been most frequently applied to AF research, further indicating that inflammation is associated with $\mathrm{AF}$, and these inflammatory markers have been described as involved in the pathogenesis of triggering and maintaining AF attacks [Sardu 2017].

In addition, another study proposes that inflammatory stimulation [eg, IFN- $\gamma$, IL-6, lipopolysaccharide (LPS), and poly(I:C) treatment] induces the overexpression of miRNA155 in breast cancer cells. This result is consistent with the idea that inflammation-induced miRNA-155 expression may contribute to inflammation-related cancers [Jiang 2010]. Recently, there is also a report regarding the strong association between inflammation and miRNA-155, which markedly promotes cell proliferation and secretion of proinflammatory markers, such as IL-6, TNF- $\alpha$, IL- $1 \beta$, and IFN- $\gamma$. In vivo studies have shown that anti-miRNA-155 administration can alleviate DSS-induced intestinal inflammation in $\mathrm{BALB} / \mathrm{c}$ mice [Lu 2017].

Our analysis demonstrated an association between miRNA155 and inflammation and found that TNF- $\alpha$, CRP, and IL-6 levels markedly increased for those recurrent patients with AF. In addition, miRNA-155 expression was positively correlated with TNF- $\alpha$, CRP, and IL- 6 in the recurrent group. These results corroborated the existence of close associations among miRNA-155, AF, and inflammation. Therefore, we speculated that the effects of intense inflammation might give rise to $\mathrm{AF}$ recurrence, further indicating that miRNA-155 might be a useful marker for evaluating AF recurrence.

$\mathrm{BNP}$ is a predictive biomarker of clinical outcomes. This has been confirmed in terms of being a cardiac mortality-predictor biomarker in cardiac and noncardiac diseases. Evidence provided by Katsanos et al showed that 6\% of elderly patients undergoing fracture surgery experienced major cardiac events postoperatively during their hospitalization. A postoperative increase in BNP levels was the most important predictor of 1-year mortality [Katsanos 2017]. Further analysis confirmed that BNP substantially improved AF risk prediction in addition to that provided by clinical factors in an independently replicated, heterogeneous population [Sinner 2014]. In our view, increased BNP levels were significantly associated with AF recurrence, and BNP levels were positively correlated with miRNA-155 levels in patients with AF recurrence. These findings demonstrated that the miRNA-155 might serve as a novel putative predictor for evaluating AF recurrence risk.

\section{Atrial Dilation and miRNA-155}

The results of a previous study have suggested that variation of pulmonary veins play a part in the maintenance of AF. Dilated pulmonary veins are associated with a high degree of stretching, and the stretch-induced arrhythmogenic activity of pulmonary veins may contribute to the initiation of AF [Chang 2007]. Atrial remodeling has been confirmed to relate to the pathogenesis of AF and is characterized by several atrial structural and functional alterations. These changes can be classified as electrical remodeling and structural remodeling. Previous reports propose that atrial enlargement is one of the components of structural remodeling. Its hallmark is atrial fibrosis [Li 1999; Allessie 2002], which affects the left atrium and is a major determinant of the progression of $\mathrm{AF}$ [Marrouche 2014]. Moreover, the presence of left atrium fibrosis is a risk factor for AF recurrence after radiofrequency catheter ablation [den Uijl 2011]. miRNA-155 has been confirmed to be closely related in pathological cardiac remodeling [Chang 2007]. Further analysis found that the loss of miRNA-155 in fibroblasts protected left ventricular function after experimental acute myocardial infarction and that the overexpression of miRNA-155 in cardiac fibroblasts was a potent contributor to cardiac remodeling [Chang 2007].

Recently, the assessment of left atrial fibrosis via 2-dimensional echocardiography-derived integrated backscatter has been validated. The value of this index in the left atrium is well correlated with the extent of collagen inside the left atrium appendage. The recent study found that patients with a large left atrium had higher values of calibrated integrated backscatter than patients with a small left atrium [den Uijl 2011]. Left atrial dilatation is an important risk factor for AF recurrence after radiofrequency catheter ablation [den Uijl 2011], and the pathological process may be related to mechanoelectrical feedback having arisen by increased stretch in the pulmonary veins and to the fact that atrial enlargement can alter atrial refractoriness and dispersion and increase vulnerability to AF [Chang 2007].

Evidence exists demonstrating that external stressors of the left atrium in AF patients can encourage cardiac architecture changes that touch off left atrial structural remodeling [Casaclang-Verzosa 2008]. The hallmark of these macroscopic cardiac architecture changes is atrial dilation [Ausma 
1997]. Significant microscopic changes include cellular hypertrophy, fibrosis, dedifferentiation, myolysis, apoptosis, and mitochondrial and sarcoplasmic reticulum disruption [Aimé-Sempé 1999; Rücker-Martin 2002; Xu 2004; Verheule 2010]. These macroscopic and microscopic changes can directly or indirectly lead to pressure or volume overload of the atria. This augmented atrial load contributes to atrial dilatation and stretching [Vaziri 1995].

In this study, our analysis suggests that the LAD in patients with $\mathrm{AF}$ is significantly greater in the recurrent group than in the nonrecurrent group, possibly indicating that the augmented atrial load might occur in patients with $\mathrm{AF}$ recurrence. Other evidence indicates the existence of significant positive correlations between miRNA-155 expression, LAD of AF patients, plasma levels of hsCRP, and AF duration. Thus, LAD, AF duration, and hsCRP levels can be considered as predictors of increased risk of AF recurrence [Marchese 2011; Cai 2013], indicating that the pathological process of AF recurrence might cause pressure or volume overload of the atria. These findings are also confirmed in our research. Our findings lead us to propose that plasma miRNA-155 levels are correlated with increased LAD in the recurrent group, with the conjecture that miRNA-155 levels might be closely related to atrial dilatation, which arouses atrial remodeling as a further direct consequence of AF.

Our analysis of the ROC curves indicates that plasma miRNA-155 levels seem to have diagnostic significance for predicting AF recurrence. On the basis of these findings, we think that miRNA-155 might be closely related to the occurrence and development of $\mathrm{AF}$, indicating that the presence of upregulation of miRNA-155 in association with inflammation might contribute to atrial remodeling in patients with AF recurrence.

\section{CONCLUSIONS}

In the current study, the presences of persistent AF and a history of MVD were related to a higher risk for AF recurrence after ablation. There were no significant differences in age, gender, duration of AF, hypertension, DM, CAD, and hyperlipidemia between the 2 groups.

Plasma miRNA-155 levels, which were closely related to LAD and the levels of CRP, BNP, as well as IL-6, were proved to play an important role in the pathological process of $\mathrm{AF}$ recurrence. Consequently, miRNA-155 levels were available for prediction of AF recurrence after cardioversion and might be an effective early warning marker and intervention target for AF patients.

\section{LIMITATIONS OF THE STUDY}

There are some limitations in the current study. First, other miRNAs may also be involved in AF pathogenesis [Sardu 2015]. Single gene research may have limitations in evaluating recurrence of AF. Second, the left atrium volume can describe atrial cardiomyopathy during AF processes [January 2014;
Kirchhof 2016; Pison 2016]. And LAD cannot reflect the higher sensitivity of the changes of left atrial function compared with left atrial volume. Third, the imaging technique can be used to study atrial fibrosis and remodeling and to predict their outcomes [Chang 2007]. Nevertheless, we used only ultrasonic diagnostic evidence in AF patients because of the high cost of MRI. Although the presence of a history of MVD was related to a higher risk for AF recurrence, the value of EF, representing the function of the left ventricle, was normal because of less population analysis in this study.

\section{ACKNOWLEDGMENTS}

Funding: This study received no financial support.

Author contributions: X.Z. and X.p.-X. performed the qRT-PCR experiments, operated the ROCHE automatic biochemical analyzer, performed the immunoenzymatic analysis, and wrote the paper. These authors also participated in the biochemical and statistical analyses and patient recruitment. X.a.-R. designed the study, performed the color Doppler ultrasonography, and participated in the ultrasonic data analysis. T.C. designed the study and participated in patient recruitment and the ultrasonic data analysis. All authors read and approved the final manuscript.

Ethics approval and consent to participate: This study was approved by the Medical Ethics Committee of the First People's Hospital of Xian Yang City and the Eighth Hospital of Xi An City; informed consent was obtained from all participants. The study was conducted in accordance with the Declaration of Helsinki.

Competing interests: The authors declare that they have no competing interests.

Consent for publication: Not applicable.

Availability of data and materials: All data generated or analyzed during this study are included in this published article. The raw data sets used and/or analyzed during the current study are available from the corresponding author (T.C.) and X.Z. on reasonable request.

Other: We thank Yi Liu from the First People's Hospital of Xian Yang City for his critical suggestions and comments on this manuscript.

\section{REFERENCES}

Aimé-Sempé C, Folliguet T, Rücker-Martin C, et al. 1999. Myocardial cell death in fibrillating and dilated human right atria. J Am Coll Cardiol 34:1577-86.

Allessie M, Ausma J, Schotten U. 2002. Electrical, contractile and structural remodeling during atrial fibrillation. Cardiovasc Res 54:230-46.

Ausma J, Wijffels M, Thoné F, Wouters L, Allessie M, Borgers M. 1997. Structural changes of atrial myocardium due to sustained atrial fibrillation in the goat. Circulation 96:3157-63.

Aviles RJ, Martin DO, Apperson-Hansen C, et al. 2003. Inflammation as a risk factor for atrial fibrillation. Circulation 108:3006-10.

Blanco RR, Austin H, Vest RN 3rd, et al. 2012. Angiotensin receptor 
type 1 single nucleotide polymorphism $1166 \mathrm{~A} / \mathrm{C}$ is associated with malignant arrhythmias and altered circulating miR-155 levels in patients with chronic heart failure. J Card Fail 18:717-23.

Cai L, Yin Y, Ling Z, et al. 2013. Predictors of late recurrence of atrial fibrillation after catheter ablation. Int J Cardiol 164:82-7.

Casaclang-Verzosa G, Gersh BJ, Tsang TS. 2008. Structural and functional remodeling of the left atrium: clinical and therapeutic implications for atrial fibrillation. J Am Coll Cardiol 51:1-11.

Chang SL, Chen YC, Chen YJ, et al. 2007. Mechanoelectrical feedback regulates the arrhythmogenic activity of pulmonary veins. Heart $93: 82-8$.

Chugh SS, Havmoeller R, Narayanan K, et al. 2014. Worldwide epidemiology of atrial fibrillation: a Global Burden of Disease 2010 Study. Circulation 129:837-47.

den Uijl DW, Delgado V, Bertini M, et al. 2011. Impact of left atrial fibrosis and left atrial size on the outcome of catheter ablation for atrial fibrillation. Heart 97:1847-51.

Fichtlscherer S, De Rosa S, Fox H, et al. 2010. Circulating microRNAs in patients with coronary artery disease. Circ Res 107:677-84.

Filipowicz W, Bhattacharyya SN, Sonenberg N. 2008. Mechanisms of post-transcriptional regulation by microRNAs: are the answers in sight? Nat Rev Genet 9:102-14.

Genovesi S, Rossi E, Gallieni M, et al. 2015. Warfarin use, mortality, bleeding and stroke in haemodialysis patients with atrial fibrillation. Nephrol Dial Transplant 30:491-8.

Hou J, Wang P, Lin L, et al. 2009. MicroRNA-146a feedback inhibits RIG-I-dependent Type I IFN production in macrophages by targeting TRAF6, IRAK1, and IRAK2. J Immunol 183:2150-8.

January CT, Wann LS, Alpert JS, et al. 2014. AHA/ACC/HRS guideline for the management of patients with atrial fibrillation: executive summary: a report of the American College of Cardiology/American Heart Association Task Force on Practice Guidelines and the Heart Rhythm Society. Circulation 130:2071-104.

Jiang S, Zhang HW, Lu MH, et al. 2010. MicroRNA-155 functions as an oncomiR in breast cancer by targeting the suppressor of cytokine signaling 1 gene. Cancer Res 70:3119-27.

Kannel WB, Benjamin EJ. 2009. Current perceptions of the epidemiology of atrial fibrillation. Cardiol Clin 27:13-24, vii.

Kannel WB, Wolf PA, Benjamin EJ, Levy D. 1998. Prevalence, incidence, prognosis, and predisposing conditions for atrial fibrillation: populationbased estimates. Am J Cardiol 82(7 suppl 1):2N-9N.

Katsanos S, Mavrogenis AF, Kafkas N, et al. 2017. Cardiac biomarkers predict 1 -year mortality in elderly patients undergoing hip fracture surgery. Orthopedics 40:e417-24.

Kirchhof P, Benussi S, Kotecha D, et al. 2016. 2016 ESC Guidelines for the management of atrial fibrillation developed in collaboration with EACTS. Eur Heart J 37:2893-962.

Li D, Fareh S, Leung TK, Nattel S. 1999. Promotion of atrial fibrillation by heart failure in dogs: atrial remodeling of a different sort. Circulation 100:87-95.

Lu ZJ, Wu JJ, Jiang WL, et al. 2017. MicroRNA-155 promotes the pathogenesis of experimental colitis by repressing SHIP-1 expression. World J Gastroenterol 23:976-85.

Marchese P, Bursi F, Delle Donne G, et al. 2011. Indexed left atrial volume predicts the recurrence of non-valvular atrial fibrillation after successful cardioversion. Eur J Echocardiogr 12:214-21.
Marfella R, Sasso FC, Siniscalchi M, et al. 2013. Brief episodes of silent atrial fibrillation predict clinical vascular brain disease in type 2 diabetic patients. J Am Coll Cardiol 62:525-30.

Marrouche NF, Wilber D, Hindricks G, et al. 2014. Association of atrial tissue fibrosis identified by delayed enhancement MRI and atrial fibrillation catheter ablation: the DECAAF study. JAMA 311:498-506.

Mitri G, Udy A, Bandeshe H, Clement P, Boots R. 2016. Variable use of amiodarone is associated with a greater risk of recurrence of atrial fibrillation in the critically ill. Crit Care 20:90.

Naccarelli GV, Varker H, Lin J, Schulman KL. 2009. Increasing prevalence of atrial fibrillation and flutter in the United States. Am J Cardiol 104:1534-9.

Nattel S, Harada M. 2014. Atrial remodeling and atrial fibrillation: recent advances and translational perspectives. J Am Coll Cardiol 63:2335-45.

O'Connell RM, Kahn D, Gibson WS, et al. 2010. MicroRNA-155 promotes autoimmune inflammation by enhancing inflammatory $\mathrm{T}$ cell development. Immunity 33:607-19.

O'Connell RM, Rao DS, Baltimore D. 2012. microRNA regulation of inflammatory responses. Annu Rev Immunol 30:295-312.

O'Connell RM, Taganov KD, Boldin MP, Cheng G, Baltimore D. 2007. MicroRNA-155 is induced during the macrophage inflammatory response. Proc Natl Acad Sci USA 104:1604-9.

Pison L, Tilz R, Jalife J, Haïssaguerre M. 2016. Pulmonary vein triggers, focal sources, rotors and atrial cardiomyopathy: implications for the choice of the most effective ablation therapy. J Intern Med 279:449-56.

Rebane A, Akdis CA. 2013. MicroRNAs: essential players in the regulation of inflammation. J Allergy Clin Immunol 132:15-26.

Rücker-Martin C, Pecker F, Godreau D, Hatem SN. 2002. Dedifferentiation of atrial myocytes during atrial fibrillation: role of fibroblast proliferation in vitro. Cardiovasc Res 55:38-52.

Sardu C, Santamaria M, Paolisso G, Marfella R. 2015. microRNA expression changes after atrial fibrillation catheter ablation. Pharmacogenomics 16:1863-77.

Sardu C, Santulli G, Santamaria M, et al. 2017. Effects of alpha lipoic acid on multiple cytokines and biomarkers and recurrence of atrial fibrillation within 1 year of catheter ablation. Am J Cardiol 119:1382-6.

Sinner MF, Stepas KA, Moser CB, et al. 2014. B-type natriuretic peptide and $\mathrm{C}$-reactive protein in the prediction of atrial fibrillation risk: the CHARGE-AF Consortium of community-based cohort studies. Europace 16:1426-33.

van Berkel TJ, Out R, Hoekstra M, Kuiper J, Biessen E, van Eck M. 2005. Scavenger receptors: friend or foe in atherosclerosis? Curr Opin Lipidol 16:525-35.

Vaziri SM, Larson MG, Lauer MS, Benjamin EJ, Levy D. 1995. Influence of blood pressure on left atrial size. The Framingham Heart Study. Hypertension 25:1155-60.

Verheule S, Tuyls E, van Hunnik A, Kuiper M, Schotten U, Allessie M. 2010. Fibrillatory conduction in the atrial free walls of goats in persistent and permanent atrial fibrillation. Circ Arrhythm Electrophysiol 3:590-9.

Wang J, Song S, Xie C, et al. 2015. MicroRNA profiling in the left atrium in patients with non-valvular paroxysmal atrial fibrillation. BMC Cardiovasc Disord 15:97.

Xu J, Cui G, Esmailian F, et al. 2004. Atrial extracellular matrix remodeling and the maintenance of atrial fibrillation. Circulation 109:363-8. 